Case Report

\title{
Cutaneous Tuberculosis- probably due to mycobacterium bovis infection: A case report
}

\section{Sanjay Kini ${ }^{1}$, Aravind Shanbhag ${ }^{2}$, Sachidananda Prabhu ${ }^{3}$}

${ }^{1}$ Assistant Professor, Department of Community Medicine, KSHEM A, Mangalore, ${ }^{2}$ Senior Resident, Department of Orthopedics, KSHEM A, M angalore, ${ }^{3}$ Assistant Professor, Department of Surgery, KSHEM A, M angalore.

*Corresponding Author : Sanjay Kini, Department of Community M edicine, KSHegde M edical Academy, Deralakatte,

Mangalore-575018, E-mail : sanjaykini2010@gmail.com

$\begin{array}{ll}\text { Received } & : 01.12 .2016 \\ \text { Review Completed } & : 25.01 .2017 \\ \text { Accepted } & : 28.01 .2017\end{array}$

Keywords: Cutaneous tuberculosis, prosthetic joint infection

\begin{tabular}{|c|}
\hline Access this article online \\
\hline Quick Response Code \\
\hline
\end{tabular}

\begin{abstract}
Cutaneous tuberculosis is an infection caused by $\mathrm{M}$. tuberculosis complex, $\mathrm{M}$. bovis and bacillus Calmette- Guérin. Depending on individual immunity, environmental factors and the type of inoculum, it may present varied clinical and evolutionary aspects. Here we found a similar case where a 63 year old female patient developed cutaneous tuberculosis either due to direct inoculation or secondary to consumption of unpasteurized milk. The wound was not healing even after several courses of antibiotics and started healing only after starting anti-tubercular treatment. Hence we recommend that clinicians should keep this possibility in mind for early diagnosisand prompt treatment.
\end{abstract}

\section{Introduction}

Cutaneoustuberculosis (TB) is essentially an invasion of the skin by Mycobacterium tuberculosis, the same bacteria that causes pulmonary tuberculosis ${ }^{1}$. In developed countries the incidence of cutaneous tuberculosis is very rare and now the incidence is also declining in developing countries $^{2-4}$. Availability of anti-tubercular treatment (ATT), improved living standards, and BCG vaccination are some of the factors contributing to this declining trend. Inhalation of airborne droplets and rarely by direct inoculation of the skin by M. tuberculosis, M . bovis, or the Bacillus calmette-guerin (BCG) are some of the routes of transmission of infection ${ }^{1}$. Tuberculosis is most often an airborne transmissible disease, but as a result of hematogenous spread or direct inoculation the cutaneous tuberculosis manifests itself in susceptible individuals. People with HIV infection, intravenous drug abusers, with diabetes mellitus, on immunosuppressive therapy, malignancies, end-stage renal disease, and infants are more prone to acquiring cutaneous tuberculosis ${ }^{5}$. Despite the recent advances in sophisticated techniques cutaneous tuberculosis is frequently elusive as it mimics a wide differential diagnosis and also evades microbiological confirmation ${ }^{6}$. Although rare, but it is important for clinicians to recognise the various clinical variants of cutaneous tuberculosis to prevent missing the diagnosis or incur a delayed diagnosis.

\section{Case Report}

A 63 year old female patient presented to the OPD at KowdoorSrinivasaHegde memorial health centre, Bailur (a satellite hospital of KSHEM A) with complaints of multiple discharging ulcers on the left leg near the ankle joint since 5 months. There were multiple pus filled abscess. There was intermittent bloody discharge from the sites of wound. There was no history of fever or trauma. The patient was non diabetic. Initially she had been treated conservatively 
by local practitioners and the wound used to heal for a short period and would recur again.

The patient then presented to our health centre for further treatment. On eliciting a detailed history we found that the patient had cattle at home and had history of consumption of unpasteurized milk. She also gave a history of undergoing Knee replacement surgery 7 months back. But there was no history suggesting prosthetic joint infection. At our health care centre she was initially put on antibiotic Tab Augmentin for 5 days, Neomycin ointment and incision \& drainage of the pus from the sites of wound was done. Since the pus was still persisting and the sites of incision and drainage had formed sinus, we referred her to NitteGajria hospital, Karkala where she was admitted and investigations were done. Blood investigations revealed total leucocyte count- 9,200/ microL, P-53, L-38, E-09, M-0, B-0. ESR- $75 \mathrm{~mm}$ at the end of $1^{\mathrm{st}} \mathrm{hr}, \mathrm{Hb}-11.7 \mathrm{~g} \%$. Patient was started on IV injAccuzon plus for 5 days. She was then discharged and followed up back at Kowdoor Srinivasa Hegde memorial health centre, Bailur and was called for alternate day dressings for almost a month.

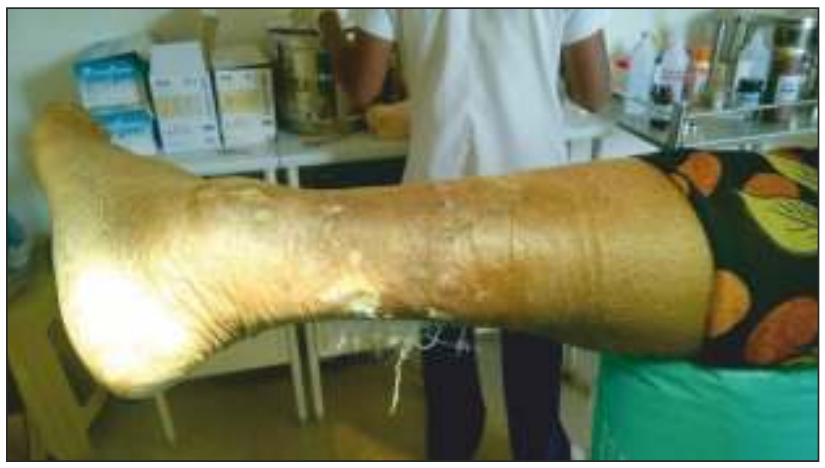

Fig 1 : M ultiple abscess on the posterior and lateral aspect of left leg

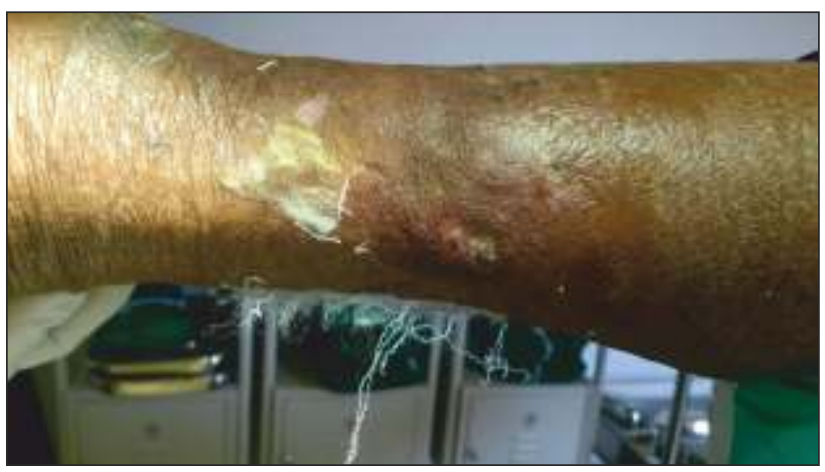

Fig 2 : Discharging sinus on the lateral aspect of left leg
Since the wound was still not healing completely, we sent the patient for excision biopsy of the ulcer. Microscopy results revealed that the section studied showed skin tissue with pseudo epitheliomatous hyperplasia. Granulation tissue with extensive necrosis surrounded by epitheloid cells, langhans giant cells, lymphocytes and plasma cells. Granulomas were extending to subcutaneous fat. Perineural infiltration was also seen. Special stain for Acid fast bacillus (AFB) and fungus was negative. But the impression from pathologist said that features are suggestive of granulomatous lesion probably due to tuberculosis.

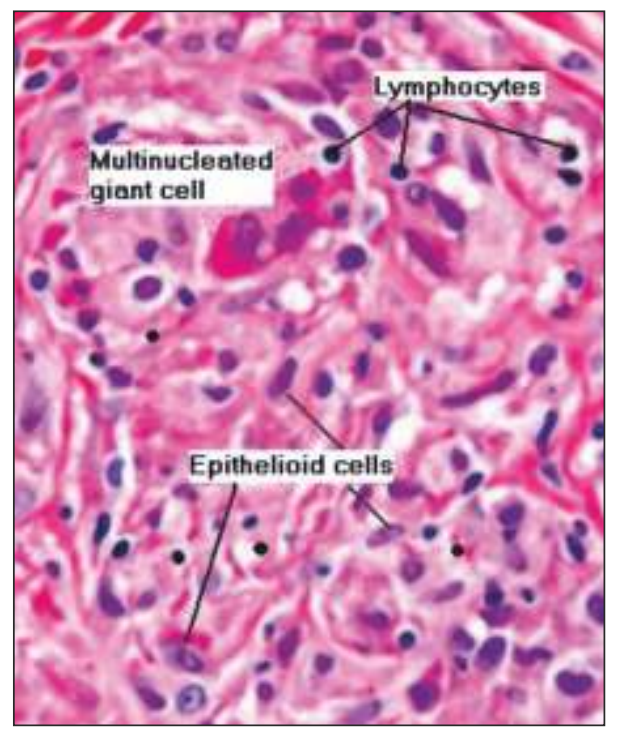

Fig 3 : Histopathology report showing multinucleated giant cells, lymphocytes and epitheloid cells

The patient was then referred to the nearby Primary Health Centre where she was started on Category I, antitubercular DOTS regimen consisting of Isoniazid $(H)$, Rifampicin (R), Pyrazinamide (Z) and Ethambutol (E), the intensive phase consisting of $2(\mathrm{HRZE})_{3}$ and continuation phase consisting of $4(\mathrm{HR})_{3}$. The wound started healing spontaneously within 2 weeks of treatment. The patient was advised to take full course for 6 months.

\section{Discussion}

Cutaneous tuberculosis can be caused by Mycobacterium tuberculosis, mycobacterium bovis and BCG vaccination. The lesions can be acquired either exogenously or endogenously, although the former one is most common. 
Although cutaneous tuberculosis is most common in underdeveloped and developing countries, the incidence is increasing even in developed countries due to infections like HIV and multi-drug resistance ${ }^{7}$. Even though the prevalence of cutaneous tuberculosis is less than 1 percent of all cases of tuberculosis, practitioners must be careful not to miss the diagnosis ${ }^{8}$.

In the present case we are suspecting thatthe patient had developed cutaneous tuberculosis due to M ycobacterium bovis because patient had history of close contact with cattle and also history of consumption of unpasteurized milk. M. bovis infects humans, causing tuberculosis through inhalation and ingestion, and by contact with mucous membranes and broken skin. Humans are spillover hosts of $M$. bovis. The clinical presentation of TB due to $M$. bovis depends on the route of infection. Oral infection acquired by drinking unpasteurized milk from diseased cattle usually results in cervical or mesenteric nodes and other forms of non-pulmonary disease. Though in our patient, we did not find lymph node enlargement, but extra-pulmonary manifestation in the form of cutaneous tuberculosis points out the possibility of infection due to M. bovis.

Cutaneous tuberculosis can also occur due to infection following knee replacement surgery. There are several studies showing tubercular infection of knee joint ${ }^{10 \cdot 12}$, but literature pertaining to tubercular infection of a replaced knee joint is scarce ${ }^{13-14}$. Some studies have shown granulomatous inflammation following joint replacement secondary to foreign body reaction ${ }^{15}$.In the present case though the patient had history of undergoing knee replacement surgery few months prior to the development of cutaneous tuberculosis, but we did not find any history which suggested infection of the prosthetic joint.

\section{References}

1. Dwari BC, Ghosh A, Paudel R, Kishore P. A clinic-epidemiological study of 50 cases of cutaneous tuberculosis in a tertiary care teaching hospital in Pokhara, Nepal. Indian J Dermatol. 2010 Jul-Sep; 55(3): 233-237.

2. Gopinathan R, Pandit D, Joshi J, Jerajani H, Mathur M. Clinical and morphological variants of cutaneous tuberculosis and its relation to mycobacterium species. IndianJ Med M icrobiol. 2001;19:193-6.

3. Kumar B, Rai R, Kaur I, Sahoo B, M uralidhar S, Radotra BD. Childhood
In the present case histopathological report showed granulation tissue with extensive necrosis surrounded by epitheloid cells, langhans giant cells, lymphocytes and plasma cells but staining for AFB was negative which indicates high immunity. Many case reports have shown similar results ${ }^{1,16}$. Since the patient responded well to antitubercular treatment retrospectively we can confirm the diagnosis of cutaneoustuberculosis.

\section{Conclusion}

Although the incidence of cutaneous tuberculosis is rare, it should be considered in patients presenting with atypical skin lesions suggestive of an underlying infectious etiology. It becomes important that physicians have a high index of suspicion in order to quickly and effectively diagnose and treat these morbid skin conditions. The present case report suggests the importance of a proper history and physical examination combined with proper laboratory and diagnostic testing in determining the etiology of a suspicious and treatment-resistant skin lesion. Prompt consideration leads to a swift diagnosis and proper treatment resulting in high patient satisfaction.

In the Indian scenario, where tuberculosis is very common, we strongly recommend pre-surgical diagnostic tests to rule out tuberculosis. We also recommend that $M$. bovis infection should be suspected in all at risk groups, particularly in rural areas.

Although the diagnosis of cutaneous tuberculosis is based on clinical features, demonstration of acid-fast bacilli on smear, tissue culture, skin biopsy, and PCR in recent years. However, in all the cases may not present with typical features and diagnosis may be dependent on clinical features, histopathological findings, and retrospective review of response to treatment.

cutaneous tuberculosis: a study over 25 years from northern India. Int J Dermatol. 2001;40:26-32.

4. Kumar B, Muralidhar S. Cutaneous tuberculosis: A-Twenty-year prospective study. Int J Tuberc Lung Dis. 1999;3:494-500.

5. Frankel A, Penrose C, Emer J. Cutaneous Tuberculosis A Practical Case Report and Review for the Dermatologist. J Clin Aesthetic Dermatol. 2009;2(10):19-27.

6. Barbagallo J, Tager $\mathrm{P}$, Ingleton $\mathrm{R}$, et al. Cutaneous tuberculosis: 
diagnosis and treatment. Am J ClinDermatol. 2002;3(5):319-328

7. Handog EB, Gabriel TG, Pineda RT. Management of cutaneous tuberculosis. DermatolTher. 2008;21(3):154-161.

8. Rai VM, Shenoi SD, Gowrinath. Tuberculous gluteal abscess coexisting with scrofuloderma and tubercular lymphadenitis. Dermatol Online J. 2005;11(3):14

9. Bilal S, Iqbal M, Murphy P, Power J. Human bovine tuberculosis remains in the differential. Journal of M edical M icrobiology 2010; 59: 1379-1382

10. Kerri O, Martini M. Tuberculosis of the knee. International Orthopedics. 1985;9:153-7.

11. Golding FC. Tuberculosis of the knee. Proc R Soc Med. 1941;34: 823-34.

12. Girdlestone GR. The pathology and treatment of tuberculosis of the knee joint. British Journal of Surgery. 2005;19:488-507

13. Al-Shaikh R, Goodman SB. Delayed-onset mycobacterium tuberculosis infection with staphylococcal superinfection after total knee replacement. AmJ Orthop. 2003;32:302-5.

14. Uppal S, Garg R. Tubercular Infection Presenting as Sinus Over Ankle Joint after Knee Replacement Surgery. J Glob Infect Dis. 2010; 2(1): 71-72.

15. Jacobs JJ, Urban RM, Wall J, Black J, Reid JD, Veneman L. Unusual foreign-body reaction to a failed total knee replacement: simulation of a sarcoma clinically and a sarcoid histologically. A case report. J Bone Joint Surg Am. 1995;77:444-51.

16. Carrega G,Bartolacci V, Burastero G, Finocchio GC, Ronca A, Riccio G. Prosthetic joint infections due to Mycobacterium tuberculosis: A report of 5 cases. Int J Surg Case Rep. 2013; 4(2): 178-181. 